\title{
RANDOM WALKS ON DIESTEL-LEADER GRAPHS
}

\author{
DANIELA BERTACCHI
}

\begin{abstract}
We investigate various features of a quite new family of graphs, introduced as a possible example of vertex-transitive graph not roughly isometric with a Cayley graph of some finitely generated group. We exhibit a natural compactification and study a large class of random walks, proving theorems concerning almost sure convergence to the boundary, a strong law of large numbers and a central limit theorem. The asymptotic type of the $n$-step transition probabilities of the simple random walk is determined.
\end{abstract}

Keywords: tree, horocyclic function, $D L$-graph, transition probabilities.

Mathematics Subject Classification: 60J15

\section{INTRODUCTION}

The subject of the present work is the study of some probabilistic features of a particular family of graphs, which are obtained by coupling two homogeneous trees: the $D L$-graphs. These graphs are named after their "inventors" Reinhard Diestel and Imre Leader, who introduced them with the aim of providing an example of a vertex-transitive graph that is not roughly isometric with a Cayley graph of some finitely generated group (see Diestel and Leader [6] for a detailed exposition of the problem; see also Woess [14] Paragraph 3.A for the definition of rough isometry between metric spaces and its relations with graph and random walk theory). This problem is still open, so that the actual aim is to collect various features of the $D L$-graphs.

$D L(p, q)$ is an induced subgraph of the cartesian product of two homogeneous trees $\mathbb{T}_{p}$ and $\mathbb{T}_{q}$ (where $p$ and $q$ are the degrees, not necessarily equal). One chooses ends $\omega_{1}$ of $\mathbb{T}_{p}$ and $\omega_{2}$ of $\mathbb{T}_{q}$ and considers the horocyclic functions $h_{1}$ and $h_{2}$ on the two trees with respect to these ends. Then $D L(p, q)$ consists of the couples $x_{1} x_{2}$ of $\mathbb{T}_{p} \times \mathbb{T}_{q}$ such that $h_{1}\left(x_{1}\right)+h_{2}\left(x_{2}\right)=0$, and $x_{1} x_{2}$ is a neighbour of $y_{1} y_{2}$ if $x_{i}$ is a neighbour of $y_{i}$ for $i=1,2$ (see Section 3).

We first deal with a wide class of random processes $\left(Z_{n}\right)_{n}$ on $D L(p, q)$ (induced by particular random walks on $\operatorname{AUT}(D L(p, q)))$. We prove that irreducible, invariant (in which sense will be clear in the sequel) random walks on $D L(p, q)$ are particular cases of these random processes. We show that under some conditions one has almost sure convergence to a natural boundary (Theorems 5.1 and 5.2), then we prove a strong law of large numbers (Theorem 6.1) and a central limit theorem (Theorems 7.1 and 7.2) for the sequence $\left(d\left(o, Z_{n}\right)\right)_{n}$ where $o$ is the starting point of the random walk and $d$ is the distance on the graph. The basic tool in the proof of these results is to study how the random walk on $D L(p, q)$ projects onto a random walk on $\mathbb{Z}$ through the horocyclic map and the use of analogous results for the homogeneous tree proved by Cartwright, Kaimanovich and Woess (see [5]).

Then we study the asymptotic decay of the transition probabilities of the simple random walk on $D L(p, q)$. More precisely, we determine its asymptotic type, and observe that, by a result of Pittet and Saloff-Coste ([10]), this coincides with the asymptotic type of any symmetric random walk on $D L(p, q)$ which is invariant under the automorphism group and has a finite second moment.

Asymptotic type is a weaker concept than asymptotic equivalence: two sequences $\left(a_{n}\right)_{n}$ and $\left(b_{n}\right)_{n}$ which are asymptotic to each other are of the same asymptotic type, but sequences of the form $\left(e^{-\lambda n} Q(n)\right)_{n}$ where $\lambda>0$ and $Q$ is a polynomial are all of asymptotic type $\left(e^{-n}\right)_{n}$. (For the definition of asymptotic type see section 8$)$. The asymptotic type of a strongly periodic random walk is the asymptotic type of the sequence $\left(p^{(n d)}(x, x)\right)_{n}$ where $d$ is the period.

The asymptotic type of the simple random walk on $D L(p, q)$ is already known in the case $p \neq q$; it is $\left(e^{-n}\right)_{n}$. We show that the asymptotic type in the case $p=q$ is $\left(e^{-n^{1 / 3}}\right)_{n}$, see Corollary 8.5. 
In Section 2 we present the construction of $D L$-graphs and other basic preliminaries. In Section 3 we state and prove three propositions which are needed in the sequel, but are also interesting in themselves: Proposition 3.1 provides an expression of the graph distance on $D L$-graphs given the distances on $\mathbb{T}_{p}$ and $\mathbb{T}_{q}$, Proposition 3.2 exhibits a natural compactification of these graphs, and Proposition 3.3 shows a useful representation of $\operatorname{AUT}(D L(p, q))$ in the case $p \neq q$. Section 4 is devoted to the definition of the class of random walks which we consider on $D L$-graphs and of the tools needed for this study. In Section 5 we study almost sure convergence to the boundary of the random walks of the family previously defined (Theorems 5.1 and 5.2). In Sections 6 and 7 we prove respectively a strong law of large numbers (Theorem 6.1) and a central limit theorem (Theorems 7.1 and 7.2) for the distance of the random walk from the origin. Finally, in Section 8 we determine the asymptotic type of the simple random walk on $D L(p, q)$ with $p=q$ (Theorem 8.4, Corollary 8.5 and Corollary 8.6).

\section{Homogeneous trees And $D L$-Graphs}

A tree is a connected graph $\mathbb{T}$ without loops or cycles. A characteristic feature of a tree is that for every pair of vertices $x, y$ there is a unique geodesic path connecting them, which we denote $\pi(x, y)$.

A homogeneous tree $\mathbb{T}$ is a tree where all vertices have the same degree: we define $\mathbb{T}_{p}$ as the homogeneous tree where each vertex has exactly $(p+1)$ neighbours. From now on we consider only homogeneous trees.

It is well known that the simple random walk on $\mathbb{T}_{p}$ is transient for every $p \geq 2$ (see, for instance, [14]).

Now we define a boundary for $\mathbb{T}$ : a geodesic ray is an infinite sequence $\left(x_{n}\right)_{n}$ of successive neighbours without repetitions. If the symmetric difference of two rays (considered as sets of vertices) has finitely many elements then the two rays are equivalent; an end is an equivalence class of rays. We denote the set of ends by $\partial \mathbb{T}$ and define $\widehat{\mathbb{T}}:=\mathbb{T} \cup \partial \mathbb{T}$ : if $\xi \in \partial \mathbb{T}$ and $x \in \mathbb{T}$ then there is a unique ray $\pi(x, \xi)$ starting at $x$ representing $\xi$. Analogously, if $\xi$ and $\eta$ are two different ends, there is a unique (bi-infinite) geodesic $\pi(\xi, \eta)$ connecting them.

We fix a reference vertex or origin $o \in \mathbb{T}$ and write $|x|=d(o, x)$ for every $x \in \mathbb{T}$. We also fix an end $\omega \in \partial \mathbb{T}$ and define the confluent with respect to $\omega$ of two elements $x, y$ of $\widehat{\mathbb{T}}$, which we denote by $x \wedge y$, as the first common vertex of the paths $\pi(x, \omega)$ and $\pi(y, \omega)$. We also denote by $\partial^{*} \mathbb{T}:=\partial \mathbb{T} \backslash\{\omega\}$.

The horocyclic function (depending on the choice of $o$ and $\omega$ ) $h: \mathbb{T} \rightarrow \mathbb{Z}$ is defined as follows:

$$
h(x):=d(x, x \wedge o)-d(o, x \wedge o)
$$

This function partitions $\mathbb{T}$ into horocycles, where the $k$-th horocycle is defined as

$$
H_{k}:=\{x \in \mathbb{T}: h(x)=k\}, \quad k \in \mathbb{Z} .
$$

Thus one can think of $\mathbb{T}$ as an "infinite genealogical tree" with $\omega$ as the "mythical ancestor" (see Cartier [4]). Then the horocycles represent successive generations and each $x \in H_{k}$ has a unique "father" in $H_{k-1}$ and $p$ "sons" in $H_{k+1}$. Then it should be clear what we mean by the $n$-th ancestor of a vertex $x$ (that is the unique vertex lying on the geodesic path $\pi(x, \omega)$ at a distance $n$ from $x)$, and, on the converse, by the $n$-th generation descending from $x$ (that is the collection of all vertices $y$ such that $d(x, y)=n$ and $x \in \pi(y, \omega)$ ). 
Figure 1: $\mathbb{T}_{2}$ in horocyclic layers

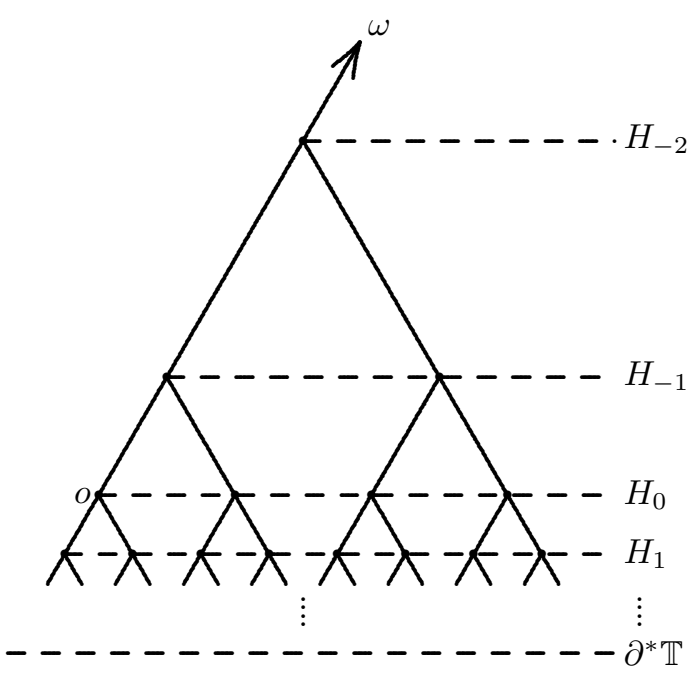

Finally, we define a metric $\theta$ on $\widehat{\mathbb{T}} \backslash\{\omega\}$, which turns out to be an ultrametric (that is $\theta(x, y) \leq$ $\max \{\theta(x, z), \theta(z, y)\}$ for all $x, y, z \in \widehat{\mathbb{T}} \backslash\{\omega\})$ :

$$
\theta(x, y):= \begin{cases}p^{-h(x \wedge y)} & \text { if } x \neq y \\ 0 & \text { if } x=y\end{cases}
$$

We will also say that a sequence $\left(x_{n}\right)_{n}$ in $\widehat{\mathbb{T}} \backslash\{\omega\}$ converges to $x \in \widehat{\mathbb{T}} \backslash\{\omega\}$ if $\theta\left(x_{n}, x\right)$ tends to zero as $n$ tends to infinity, and that it converges to $\omega$ if $\theta\left(x_{n}, o\right)$ tends to infinity. (Note that $\theta$ is not topologically equivalent to the natural distance $d$ ). We recall that this notion of convergence is the same induced by the ultrametric defined on $\widehat{\mathbb{T}}$ using confluents with respect to the origin (namely the ultrametric $\theta$ defined in [5] Section 2.A).

We now define the Diestel-Leader graphs (see also [6]). $D L(p, q)$ is an induced subgraph of the cartesian product of two homogeneous trees $\mathbb{T}^{1}=\mathbb{T}_{p}$ and $\mathbb{T}^{2}=\mathbb{T}_{q}$ ( $p$ and $q$ not necessarily equal). We fix an origin (respectively $o_{1}$ and $o_{2}$ ) and an end (respectively $\omega_{1}$ and $\omega_{2}$ ) in each tree and consider the horocyclic functions $h_{1}: \mathbb{T}^{1} \rightarrow \mathbb{Z}$ and $h_{2}: \mathbb{T}^{2} \rightarrow \mathbb{Z}$ thus determined. Then

$$
D L(p, q):=\left\{x_{1} x_{2} \in \mathbb{T}^{1} \times \mathbb{T}^{2}: h_{1}\left(x_{1}\right)+h_{2}\left(x_{2}\right)=0\right\}
$$

with the following neighbourhood relation: $x_{1} x_{2} \sim y_{1} y_{2}$ if and only if $x_{i} \sim y_{i}$ for $i=1,2$.

To visualize $D L(p, q)$, draw $\mathbb{T}^{1}$ as in Figure 1 and on its right $\mathbb{T}^{2}$ in the same way, but upside down, with the respective horocycles $H_{k}\left(\mathbb{T}^{1}\right)$ and $H_{-k}\left(\mathbb{T}^{2}\right)$ on the same level. Imagine that the two origins are connected by an infinitely elastic spring which can move along the two trees, but has to remain horizontal. Then from $o_{1} o_{2} \in D L(p, q)$ one may move upwards to the father of $o_{1}$ and to one of the sons of $o_{2}$, or downwards analogously, reaching one of the neighbours of $o_{1} o_{2}$ (which we will briefly call $o$ ). Similarly one can catch the idea of the simple random walk on $D L(p, q)$. 


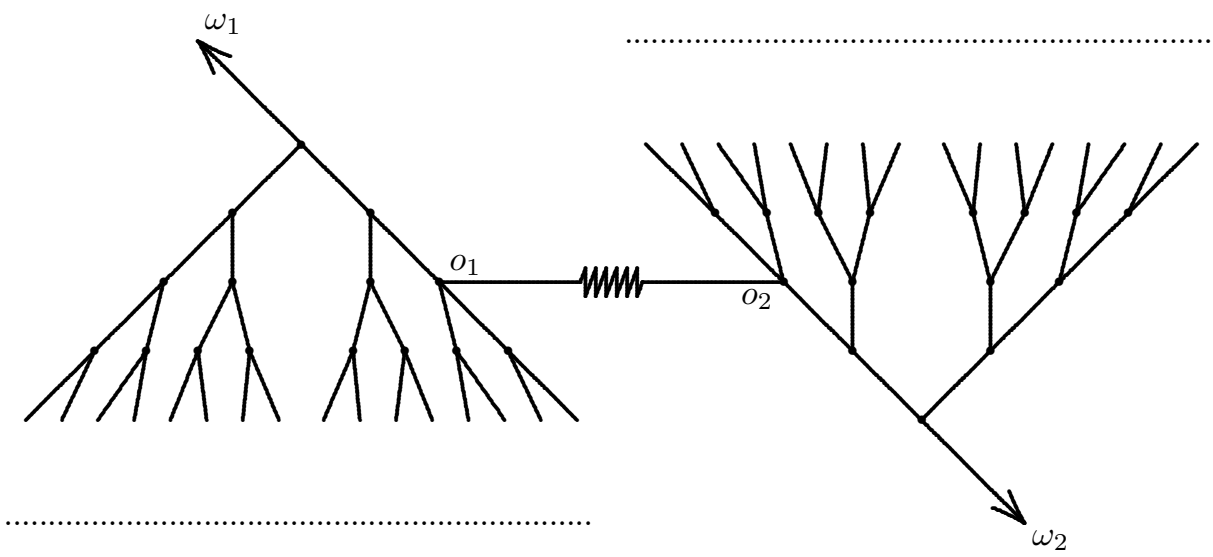

Figure 2: the representation of $D L(2,2)$

\section{Metrics AND AUtOMORPhisms ON $D L$-GRAPHS}

The $D L$-graphs are endowed with the natural graph distance:

$$
d(x, y)=\min \left\{n: \exists x_{0}, \ldots, x_{n} \in D L(p, q) \text { and } x=x_{0} \sim x_{1} \sim \cdots \sim x_{n}=y\right\} .
$$

The following proposition will be useful in the sequel, but is interesting in itself, as it links graph distance on $D L(p, q)$ with graph distances on $\mathbb{T}^{1}$ and $\mathbb{T}^{2}$ and the horocyclic function.

Proposition 3.1. Let $x_{1} x_{2}$ and $y_{1} y_{2}$ be two elements of $D L(p, q)$. Then

$$
d\left(y_{1} y_{2}, x_{1} x_{2}\right)=d_{1}\left(y_{1}, x_{1}\right)+d_{2}\left(y_{2}, x_{2}\right)-\left|h_{1}\left(y_{1}\right)-h_{1}\left(x_{1}\right)\right| .
$$

Proof. We first note that it is sufficient to prove the thesis in the case that $y_{1} y_{2}=o_{1} o_{2}=o$. Recall that, if we define $k_{i}:=d_{i}\left(x_{i}, x_{i} \wedge o_{i}\right)$ and $l_{i}:=d_{i}\left(x_{i} \wedge o_{i}, o_{i}\right)(i=1,2)$, then

$$
\begin{aligned}
d_{i}\left(o_{i}, x_{i}\right) & =k_{i}+l_{i}, \\
h_{i}\left(x_{i}\right) & =k_{i}-l_{i} .
\end{aligned}
$$

We distinguish three cases and in each of them exhibit a path with minimal length.

(a) If $h_{1}\left(x_{1}\right)<0$ choose a path divided into two parts: $o_{1} o_{2} \rightarrow \bar{x} x_{2} \rightarrow x_{1} x_{2}$, where $\bar{x} \in \mathbb{T}^{1}$ is such that $\bar{x}=\bar{x} \wedge o_{1}$ and $h_{1}(\bar{x})=h_{1}\left(x_{1}\right)$ (that is $\bar{x}$ is the ancestor of the origin on the same horocycle as $\left.x_{1}\right)$. The projection of the first part of this path onto $\mathbb{T}^{2}$ is the shortest path from $o_{2}$ to $x_{2}$ (whose length is $l_{2}+k_{2}$ ), its projection onto $\mathbb{T}^{1}$ may be any path descending from $o_{1}$ to any of the origin's sons at distance $l_{2}$ and then climbing back to the origin and then to $\bar{x}$. The projection of the second part of the path onto $\mathbb{T}^{1}$ is a path going from $\bar{x}$ to $x_{1} \wedge o_{1}$ (whose length is $l_{1}-\left|h_{1}\left(x_{1}\right)\right|$ ) plus a path descending from $x_{1} \wedge o_{1}$ to $x_{1}$ (whose length is $k_{1}$ ). Its projection onto $\mathbb{T}^{2}$ may be any path descending from $x_{2}$ to any of its sons and then climbing back to $x_{2}$. The described path has total length $d_{1}\left(o_{1}, x_{1}\right)+d_{2}\left(o_{2}, x_{2}\right)-\left|h_{1}\left(x_{1}\right)\right|$ and any other path from $o_{1} o_{2}$ to $x_{1} x_{2}$ will be at least as long. This concludes the proof in this first case.

(b) If $h_{1}\left(x_{1}\right)=0$ choose a minimal path leading from $o_{1} o_{2}$ to $x_{1} o_{2}$ and then to $x_{1} x_{2}$ : its length will be equal to $d_{1}\left(o_{1}, x_{1}\right)+d_{2}\left(o_{2}, x_{2}\right)$ and will be minimal.

(c) If $h_{1}\left(x_{1}\right)>0$ the choice of the path is analogous with the one we explained in case (a), once we reverse the roles played by $\mathbb{T}^{1}$ and $\mathbb{T}^{2}$ : the path will lead from $o_{1} o_{2}$ to $x_{1} \bar{x}$ and then to $x_{1} x_{2}$, where $\bar{x} \in \mathbb{T}^{2}$ is the ancestor of the origin on the same horocycle as $x_{2}$.

Using the ultrametric defined on homogeneous trees, we can define a metric $\Theta$ on $D L(p, q)$ (which is not an ultrametric):

$$
\Theta\left(x_{1} x_{2}, y_{1} y_{2}\right):=\theta_{1}\left(x_{1}, y_{1}\right)+\theta_{2}\left(x_{2}, y_{2}\right)
$$


where $\theta_{i}$ is the ultrametric defined on $\widehat{\mathbb{T}}^{i} \backslash\left\{\omega_{i}\right\}, i=1,2$ (see Section 2). Moreover we say that a sequence $\left(x_{n}^{1} x_{n}^{2}\right)_{n} \subset D L(p, q)$ converges to $\xi^{1} \xi^{2}$, where $\xi^{i} \in \widehat{\mathbb{T}}^{i}, i=1,2$, if and only if $\left(x_{n}^{i}\right)_{n}$ converges to $\xi^{i}, i=1,2$ (according to the definition of Section 2). We note that if the limit $\xi^{1} \xi^{2}$ is such that $\xi^{i} \neq \omega_{i}, i=1,2$, then we have convergence with respect to the metric $\Theta$.

There is a natural compactification $\widehat{D L}(p, q)$ of $D L(p, q)$, obtained as the closure of $D L(p, q)$ in $\widehat{\mathbb{T}}^{1} \times \widehat{\mathbb{T}}^{2}$.

\section{Proposition 3.2.}

$$
\widehat{D L}=\left(\left\{\omega_{1}\right\} \times \widehat{\mathbb{T}}^{2}\right) \cup\left(\widehat{\mathbb{T}}^{1} \times\left\{\omega_{2}\right\}\right) .
$$

Proof. Given a sequence $\left(x_{n}^{1} x_{n}^{2}\right)_{n} \subset D L(p, q)$, we may look at the two sequences $\left(x_{n}^{1}\right)_{n} \subset \mathbb{T}^{1}$ and $\left(x_{n}^{2}\right)_{n} \subset \mathbb{T}^{2}$ separately, keeping in mind that $h_{1}\left(x_{n}^{1}\right)+h_{2}\left(x_{n}^{2}\right)=0$ for all $n \in \mathbb{N}$.

We suppose that $d\left(o_{1} o_{2}, x_{n}^{1} x_{n}^{2}\right)$ tends to infinity and that $\left(x_{n}^{i}\right)_{n}$ converges to an element of $\widehat{\mathbb{T}}^{i}$, for $i=1,2$.

For sequences in $\mathbb{T}^{i}$, we distinguish between the three following limits: $\omega_{i}, \xi^{i} \in \partial^{*} \mathbb{T}^{i}, x^{i} \in \mathbb{T}^{i}(i=1,2)$.

There are only five possible limits: $\left(\omega_{1}, \xi^{2}\right),\left(\xi^{1}, \omega_{2}\right),\left(\omega_{1}, \omega_{2}\right),\left(\omega_{1}, x^{2}\right),\left(x^{1}, \omega_{2}\right)$. We provide an example for each:

(1) $x_{n}^{1} \rightarrow \omega_{1}$ and $x_{n}^{2} \rightarrow \xi^{2} \in \partial^{*} \mathbb{T}^{2}$ : for instance let $x_{n}^{2}$ be the vertex in $\pi\left(o_{2}, \xi^{2}\right)$ at a distance $n$ from $o_{2}$, and let $j=h_{2}\left(x_{n}^{2}\right)$. Now let $x_{n}^{1}$ be the $j$-th ancestor of $o_{1}$ if $j>0, x_{n}^{1}=o_{1}$ if $j=0$ and let $x_{n}^{1}$ be one of the $(-j)$-th descendants of $o_{1}$ if $j<0$;

(2) $x_{n}^{1} \rightarrow \xi^{1} \in \partial^{*} \mathbb{T}^{1}$ and $x_{n}^{2} \rightarrow \omega_{2}$ : the example is constructed as in the preceding case, but with the roles of $\mathbb{T}^{1}$ and $\mathbb{T}^{2}$ reversed;

(3) $x_{n}^{1} \rightarrow \omega_{1}$ and $x_{n}^{2} \rightarrow \omega_{2}$ : for instance, let $x_{n}^{i} \in H_{0}^{i}$ for $i=1$, 2, with $d_{i}\left(o_{i}, x_{n}^{i}\right) \rightarrow+\infty$;

(4) $x_{n}^{1} \rightarrow \omega_{1}$ and $x_{n}^{2} \rightarrow x^{2} \in \mathbb{T}^{2}$ : for instance, let $x_{n}^{2}=x^{2}$ for all $n \in \mathbb{N}$, and let $j=h_{2}\left(x^{2}\right)$. Then choose $x_{n}^{1} \in H_{-j}^{1}$, with $d_{1}\left(o_{1}, x_{n}^{1}\right) \rightarrow+\infty$;

(5) $x_{n}^{1} \rightarrow x^{1} \in \mathbb{T}^{1}$ and $x_{n}^{2} \rightarrow \omega_{2}$ : the example is constructed as in the preceding case, but with the roles of $\mathbb{T}^{1}$ and $\mathbb{T}^{2}$ reversed.

In other words, it is impossible that

(a) $x_{n}^{1} \rightarrow \xi^{1} \in \partial^{*} \mathbb{T}^{1}$ and $x_{n}^{2} \not \omega_{2}$ or $x_{n}^{2} \rightarrow \xi^{2} \in \partial^{*} \mathbb{T}^{2}$ and $x_{n}^{1} \nrightarrow \omega_{1}$. In fact, if $x_{n}^{1} \rightarrow \xi^{1}$ then $h_{1}\left(x_{n}^{1}\right) \rightarrow+\infty$ and then $h_{2}\left(x_{n}^{2}\right) \rightarrow-\infty$ and this forces $x_{n}^{2}$ to tend to $\omega_{2}$;

(b) $x_{n}^{1} \rightarrow x^{1} \in \mathbb{T}^{1}$ and $x_{n}^{2} \nrightarrow \omega_{2}$ or $x_{n}^{2} \rightarrow x^{2} \in \mathbb{T}^{2}$ and $x_{n}^{1} \nrightarrow \omega_{1}$. In fact, if $x_{n}^{2} \rightarrow x^{2} \in \mathbb{T}^{2}$ then $d\left(o_{1} o_{2}, x_{n}^{1} x_{n}^{2}\right) \nrightarrow+\infty$; on the other hand, if $x_{n}^{2} \rightarrow \xi^{2} \in \partial^{*} \mathbb{T}^{2}$ then $h_{2}\left(x_{n}^{2}\right) \rightarrow-\infty$, in contradiction with the hypothesis that $h_{2}\left(x_{n}^{2}\right)=-h_{1}\left(x^{1}\right)$ for all sufficiently large $n$.

Finally, we present a useful representation for the automorphism group $\operatorname{AUT}(D L(p, q))$ in the case $p \neq q$. This representation was first observed by O. Schramm (personal communication to W. Woess); here, we give our own proof. We recall that, given a homogeneous tree $\mathbb{T}$ with a fixed end $\omega$, its affine group is the group $\operatorname{AFF}(\mathbb{T})$ of all automorphisms $\gamma \in \operatorname{AUT}(\mathbb{T})$ which fix $\omega$ (note that a different choice of $\omega$ merely corresponds to a choice of a conjugate of $\operatorname{AFF}(\mathbb{T}))$. We note that

$$
\gamma(\pi(x, \omega))=\pi(\gamma x, \omega), \quad \forall \gamma \in \operatorname{AFF}(\mathbb{T}), x \in \mathbb{T} .
$$

Now let $\Gamma_{1}=\operatorname{AFF}\left(\mathbb{T}^{1}\right)$ and $\Gamma_{2}=\operatorname{AFF}\left(\mathbb{T}^{2}\right)$ (with respect to $\omega_{1}$ and $\omega_{2}$ respectively): the following proposition holds.

Proposition 3.3. If $p \neq q$ then $\operatorname{AUT}(D L(p, q))$ coincides with the group

$$
\Gamma:=\left\{\gamma_{1} \gamma_{2} \in \Gamma_{1} \times \Gamma_{2}: h_{1}\left(\gamma_{1} o_{1}\right)+h_{2}\left(\gamma_{2} o_{2}\right)=0\right\} .
$$

Proof. It is obvious that $\Gamma$ is a subgroup of $\operatorname{AUT}(D L(p, q))$. Now we construct an injective group homomorphism $f: \operatorname{AUT}(D L(p, q)) \mapsto \Gamma$ and this will prove our thesis.

If $\gamma \in \operatorname{AUT}(D L(p, q))$ then $f(\gamma)=\gamma_{1} \gamma_{2}$ where $\gamma_{i}$ is defined as follows:

$$
\gamma_{i} x_{i}=y_{i}, \quad i=1,2,
$$

whenever $\gamma\left(x_{1} x_{2}\right)=y_{1} y_{2}$. 
In order to prove that $f$ is well defined, we prove that it is impossible that, given a fixed $\gamma \in \operatorname{AUT}(D L(p, q))$, $\gamma\left(x_{1} x_{2}\right)=y_{1} y_{2}$ and $\gamma\left(x_{1} \bar{x}_{2}\right)=z_{1} z_{2}$ with $y_{1} \neq z_{1}$ (in which case $\gamma_{1}$ would not be well defined - we focus on the first component, but the argument applies to the second component as well).

Suppose that $\gamma\left(x_{1} x_{2}\right)=y_{1} y_{2}$ and $\gamma\left(x_{1} \bar{x}_{2}\right)=z_{1} z_{2}$ : since $x_{2}$ and $\bar{x}_{2}$ lie on the same horocycle in $\mathbb{T}^{2}$, their distance is even, let $d_{2}\left(x_{2}, \bar{x}_{2}\right)=2 k$. Then there are exactly $p^{k}$ distinct geodesic paths between $x_{1} x_{2}$ and $x_{1} \bar{x}_{2}$, and each of them must be mapped by $f$ to a distinct geodesic path between $y_{1} y_{2}$ and $z_{1} z_{2}$ (and viceversa, since $\gamma^{-1}$ is an automorphism too). We distinguish between two cases:

(a) if $y_{2}=z_{2}$ then $y_{1}$ and $z_{1}$ lie on the same horocycle in $\mathbb{T}^{1}$ and

$$
2 k=d\left(y_{1} y_{2}, z_{1} z_{2}\right)=d_{1}\left(y_{1}, z_{1}\right) .
$$

Hence there are $q^{k}$ distinct geodesic paths between $y_{1} y_{2}$ and $z_{1} z_{2}$, but then it should be $p^{k}=q^{k}$, which contradicts our hypothesis that $p \neq q$.

(b) if $y_{2} \neq z_{2}$ we distinguish between two subcases:

(b1) if $y_{1}$ and $z_{1}$ lie on the same horocycle: then

$$
d_{1}\left(y_{1}, z_{1}\right)=2 n, \quad d_{2}\left(y_{2}, z_{2}\right)=2 m,
$$

where $n, m \in \mathbb{N}$ and $2 k=2 n+2 m$. In this case there are $p^{m} q^{n}$ geodesic paths between $y_{1} y_{2}$ and $z_{1} z_{2}$, then it should be $p^{n} p^{m}=p^{m} q^{n}$, whence $p=q$, again a contradiction.

(b2) if $y_{1}$ and $z_{1}$ do not lie on the same horocycle:

$$
\begin{aligned}
& d_{1}\left(y_{1}, z_{1}\right)=\left|h_{1}\left(y_{1}\right)-h_{1}\left(z_{1}\right)\right|+2 n=: h+2 n, \\
& d_{2}\left(y_{2}, z_{2}\right)=h+2 m, \\
& 2 k=d\left(y_{1} y_{2}, z_{1} z_{2}\right)=2 n+2 m+h,
\end{aligned}
$$

where $h, m, n, k \in \mathbb{N}$. Without loss of generality we can suppose that $m \leq n$ : then there are $p^{m} q^{n}$ geodesic paths between $y_{1} y_{2}$ and $z_{1} z_{2}$, as in the preceding case, whence the same contradiction.

It is easy to see that $\gamma_{1}$ and $\gamma_{2}$ are automorphisms of $\mathbb{T}^{1}$ and $\mathbb{T}^{2}$ which fix $\omega_{1}$ and $\omega_{2}$ respectively, and that $h_{1}\left(\gamma_{1} o_{1}\right)+h_{2}\left(\gamma_{2} o_{2}\right)=0$. Finally, given $\gamma_{1} \gamma_{2} \in \Gamma$ there exists only one $\gamma \in \operatorname{AUT}(D L(p, q))$ such that $f(\gamma)=\gamma_{1} \gamma_{2}$, namely $\gamma\left(x_{1} x_{2}\right)=\gamma_{1}\left(x_{1}\right) \gamma_{2}\left(x_{2}\right)$ and $f$ is injective.

\section{INDUCED PROCESSES ON DL-GRAPHS}

In the sequel we will study a rather general kind of random walks on $D L$-graphs. We start with some definitions, first recalling some features of homogeneous trees (see Cartwright, Kaimanovich and Woess [5]) and then introducing their analogs on $D L$-graphs.

Observe that $\operatorname{AFF}(\mathbb{T})$ maps horocycles onto horocycles and

$$
h\left(\gamma x_{1}\right)-h\left(\gamma x_{2}\right)=h\left(x_{1}\right)-h\left(x_{2}\right) \quad \forall \gamma \in \operatorname{AFF}(\mathbb{T}), x_{1}, x_{2} \in \mathbb{T} .
$$

Hence the mapping

$$
\bar{\Phi}: \operatorname{AFF}(\mathbb{T}) \rightarrow \mathbb{Z}, \quad \gamma \mapsto h(\gamma o)
$$

is a group homomorphism such that $\gamma H_{k}=H_{k+\bar{\Phi}(\gamma)}$ for every $k \in \mathbb{Z}$, and represents the "drift" of $\gamma$ on the horocycles.

We define horocyclic group $\operatorname{HOR}(\mathbb{T})$ the kernel of $\bar{\Phi}$, that is the subset of $\operatorname{AFF}(\mathbb{T})$ which preserves any horocycle (as a set).

We say that a subgroup $\Delta$ of $\operatorname{AFF}(\mathbb{T})$ is exceptional if $\Delta \subset \operatorname{HOR}(\mathbb{T})$ or if it fixes an element of $\partial^{*} \mathbb{T}$.

Now let $\mu$ be a Borel probability measure on $\Gamma$, and let $\mu_{1}$ and $\mu_{2}$ be its projections on $\Gamma_{1}$ and $\Gamma_{2}$, that is

$$
\begin{cases}\mu_{1}(A)=\mu\left(A \times \Gamma_{2}\right), & A \in \mathcal{B}\left(\Gamma_{1}\right) \\ \mu_{2}(B)=\mu\left(\Gamma_{1} \times B\right), & B \in \mathcal{B}\left(\Gamma_{2}\right)\end{cases}
$$

where $\mathcal{B}\left(\Gamma_{i}\right)$ is the family of the Borel sets of $\Gamma_{i}, i=1,2$.

Basic assumption: we assume that the closed subgroups generated in $\Gamma_{1}$ and $\Gamma_{2}$ respectively by the support of $\mu_{1}$ and $\mu_{2}$ are non-exceptional. Here, "closed" refers to the topology of pointwise convergence on $\operatorname{AUT}(\mathbb{T})$. 
Let $\left(X_{n}\right)_{n}$ be a sequence of i.i.d. $\Gamma$-valued random variables with distribution $\mu$. The right random walk on $\Gamma$ with law $\mu$ is the sequence of random variables

$$
R_{0}=\mathrm{id}, \quad R_{n}=\mathrm{id} X_{1} \cdots X_{n}
$$

where id is the identity in $\Gamma$. This random walk induces two random walks, on $\Gamma_{1}$ and $\Gamma_{2}$ respectively, which we call $\left(R_{n}^{1}\right)_{n}$ and $\left(R_{n}^{2}\right)_{n}$. Furthermore, $\left(R_{n}\right)_{n}$ induces processes on $D L(p, q), \mathbb{T}^{1}$ and $\mathbb{T}^{2}$ respectively: $\left(R_{n} O\right)_{n},\left(R_{n}^{1} O_{1}\right)_{n}$ and $\left(R_{n}^{2} O_{2}\right)_{n}$. It is worth noting that in general $\left(R_{n}^{i} O_{i}\right)_{n}(i=1,2)$ is not a Markov chain, but has independent distance increments (see [5]). Moreover, by the same arguments as in [5] Section 3.A, $\left(R_{n}^{1}\right)_{n}$ and $\left(R_{n}^{2}\right)_{n}$ both are transient (that is with probability one they leave any compact set after finite time), hence so is $\left(R_{n}\right)_{n}$.

Next we define

$$
\begin{array}{ll}
\Phi: \quad & \Gamma \mapsto \mathbb{Z}, \\
& \gamma_{1} \gamma_{2} \mapsto h_{1}\left(\gamma_{1} o_{1}\right)
\end{array}
$$

which represents a projection of the random walk on $\Gamma$ (and then also of the induced process on $D L(p, q)$ ) onto a random walk on $\mathbb{Z}$. Note that if we put $\Phi_{i}\left(\gamma_{i}\right)=\bar{\Phi}\left(\gamma_{i}\right), i=1,2$, then

$$
\Phi\left(\gamma_{1} \gamma_{2}\right)=\Phi_{1}\left(\gamma_{1}\right)=-\Phi_{2}\left(\gamma_{2}\right) .
$$

From equation 1 it follows that $\Phi$ is a group homomorphism.

We define the absolute moments of $\mu$ :

$$
m_{r}(\mu):=\mathbb{E}\left(\left|X_{1}\right|^{r}\right)=\int_{\Gamma} d(o, \gamma o)^{r} \mu(\mathrm{d} \gamma)
$$

where $r>0$ and $|\gamma|:=d(o, \gamma o)$. Moreover, let $\Phi(\mu)$ be the image of $\mu$ on $\mathbb{Z}$ :

$$
\Phi(\mu)(k)=\mu\left(\Phi^{-1}(\{k\})\right)=\mu\left(\left\{\gamma: h_{1}\left(\gamma_{1} o_{1}\right)=k\right\}\right) .
$$

Then $\Phi\left(R_{n}\right)=\Phi\left(X_{1}\right)+\cdots+\Phi\left(X_{n}\right)$, since $\Phi$ is a group homomorphism and by the definition of $R_{n}$, that is $\Phi\left(R_{n}\right)$ is the sum of $n$ i.i.d. random variables $\Phi\left(X_{i}\right)$ with law $\Phi(\mu)$.

If $\Phi(\mu)$ has finite first absolute moment, we define the mean drift as

$$
\operatorname{MEAN}(\Phi(\mu)):=\sum_{k \in \mathbb{Z}} k \Phi(\mu)(k)=\int_{\Gamma} \Phi(\gamma) \mu(\mathrm{d} \gamma)
$$

By equation 3 we get that whenever $m_{1}(\Phi(\mu))$ exists and is finite we have

$$
\operatorname{MEAN}(\Phi(\mu))=\operatorname{MEAN}\left(\Phi_{1}\left(\mu_{1}\right)\right)=-\operatorname{MEAN}\left(\Phi_{2}\left(\mu_{2}\right)\right)
$$

and whenever $m_{2}(\Phi(\mu))$ exists and is finite then

$$
\operatorname{VAR}(\Phi(\mu))=\operatorname{VAR}\left(\Phi_{1}\left(\mu_{1}\right)\right)=\operatorname{VAR}\left(\Phi_{2}\left(\mu_{2}\right)\right),
$$

where $\operatorname{VAR}(\cdot)$ denotes variance.

Remark 4.1. Even if the induced processes $\left(R_{n} O\right)_{n}$ are not, in general, Markov chains, any irreducible, $\Gamma$-invariant random walk on $D L(p, q)$ can be viewed as a particular case of such processes, satisfying our basic assumption, which implies that the results of Sections 5, 6, 7, apply to these random walks. In fact, the following proposition holds.

Proposition 4.2. Given an irreducible, $\Gamma$-invariant random walk $\left(Z_{n}\right)_{n}$ on $D L(p, q)$, there exists a Borel probability measure $\mu$ on $\Gamma$, satisfying our basic assumption, and such that if $\left(S_{n}\right)_{n}$ is the induced right random walk on $\Gamma,\left(S_{n} O\right)_{n}$ is a model of the random walk $\left(Z_{n}\right)_{n}$ starting at $o$.

Proof. Following Woess [13], Section 3, we construct a Borel probability measure $\mu$ on $\Gamma$ :

$$
\mu(\mathrm{d} \gamma)=p(o, \gamma o) \mathrm{d} \gamma
$$

where $\mathrm{d} \gamma$ is the left Haar measure on $\Gamma$ such that $\int_{\Gamma_{o}} \mathrm{~d} \gamma=1\left(\Gamma_{o}\right.$ is the subgroup of $\Gamma$ which fixes $o$ ), and $\{p(x, y): x, y \in D L(p, q)\}$ is the set of the transition probabilities of $\left(Z_{n}\right)_{n}$. (For further details about integration on locally compact groups and the properties of the Haar measure, see Hewitt and Ross [8] or Woess [14]). 
Thus we can define a sequence of independent random variables $\left(X_{n}\right)_{n}$ distributed according to $\mu$ and the right random walk on $\Gamma$ as

$$
S_{n}=\mathrm{id} \cdot X_{1} \cdot X_{2} \cdots X_{n}
$$

Then Lemma 3.1 in [13] states that $\left(S_{n} O\right)_{n}$ is a model of the random walk $\left(Z_{n}\right)_{n}$ starting at $o$.

Hence in order to prove our thesis we only need to show that if $\mu_{1}$ and $\mu_{2}$ are the projections of $\mu$ as defined in equation 2 , then the closed subgroups generated in $\Gamma_{1}$ and $\Gamma_{2}$ respectively by the support of $\mu_{1}$ and $\mu_{2}$ are non-exceptional. But this is a consequence of the hypothesis of irreducibility.

Let us suppose that the closed subgroup generated by the support of $\mu_{1}$, which we call $C_{1}$, fixes the horocycles, that is for all $\gamma_{1} \in C_{1}, h_{1}\left(\gamma_{1} o_{1}\right)=0$. We prove that in this case $p\left(o, x_{1} x_{2}\right)=0$ if $x_{1} \notin H_{0}^{1}$, whence from any vertex of $D L(p, q)$, with a finite number of steps, we can reach only vertices on the same horocyclic level, in contradiction with irreducibility. Suppose that $p\left(o, y_{1} y_{2}\right) \neq 0$ with $y_{1} y_{2} \notin H_{0}^{1}$ : then $A:=\left\{\gamma: \gamma_{O}=y_{1} y_{2}\right\}$ is an open set in $\Gamma$ (with respect to the topology of pointwise convergence), hence has positive Haar measure $|A|$. Thus

$$
\mu(A)=\int_{A} p(o, \gamma o) \mathrm{d} \gamma>0 .
$$

Moreover, let $C=\overline{\text { supp } \mu}: C \subset C_{1} \times \Gamma_{2} ; \mu(A) \leq \mu(A \cap C)=\mu(\emptyset)=0$, a contradiction.

Now suppose that $C_{1}$ fixes an end $\xi^{1}$ : then it fixes $\pi\left(o_{1}, \xi^{1}\right)$ as a set. In this case we prove that $p\left(z_{1} z_{2}, x_{1} x_{2}\right)=0$ if $z_{1} \in \pi\left(o_{1}, \xi^{1}\right)$ and $x_{1} \notin \pi\left(o_{1}, \xi^{1}\right)$, whence whence from any vertex of $\pi\left(o_{1}, \xi^{1}\right)$, with a finite number of steps, we can reach only vertices with the first component in $\pi\left(o_{1}, \xi^{1}\right)$, in contradiction with irreducibility. It suffices to prove that $p\left(o, x_{1} x_{2}\right)=0$ if $x_{1} \notin \pi\left(o_{1}, \xi^{1}\right)$. If there exists $y_{1} y_{2}$ such that $p\left(o, y_{1} y_{2}\right) \neq 0$ and $y_{1} \notin \pi\left(o_{1}, \xi^{1}\right)$, then $\mu(A)=\mu\left(\left\{\gamma: \gamma o=y_{1} y_{2}\right\}\right)>0$, whereas $\mu(A) \leq \mu(A \cap C)=\mu(\emptyset)=0$, again a contradiction.

\section{Convergence of $R_{n} O$}

We study convergence of the sequence $\left(R_{n} O\right)_{n}$ with respect to the metric $\Theta$ on $D L(p, q)$. We note that, since the random walk $\left(R_{n}\right)_{n}$ is transient, $d\left(o, R_{n} o\right)$ tends to infinity almost surely. We first deal with the case of nonzero mean drift.

Theorem 5.1. (a) If $m_{1}(\mu)<\infty$ and $\operatorname{MEAN}(\Phi(\mu))<0$, then $R_{n} o \rightarrow\left(\omega_{1}, R_{\infty}^{2}\right)$ almost surely, where $R_{\infty}^{2}$ is a random element of $\partial^{*} \mathbb{T}^{2}$.

(b) If $m_{1}(\mu)<\infty$ and MEAN $(\Phi(\mu))>0$, then $R_{n} O \rightarrow\left(R_{\infty}^{1}, \omega_{2}\right)$ almost surely, where $R_{\infty}^{1}$ is a random element of $\partial^{*} \mathbb{T}^{1}$.

Proof. (a) From the hypotheses we get MEAN $\left(\Phi_{1}\left(\mu_{1}\right)\right)<0$ and MEAN $\left(\Phi_{2}\left(\mu_{2}\right)\right)>0$. Then by [5] Theorem 2 we have that $R_{n}^{1} o_{1} \rightarrow \omega_{1}$ almost surely and $\left(R_{n}^{2} o_{2}\right)_{n}$ converges almost surely to a random element of $\partial^{*} \mathbb{T}^{2}$.

(b) The proof is the same as in (a), with the roles of $\mathbb{T}^{1}$ and $\mathbb{T}^{2}$ reversed.

We note that in the case MEAN $(\Phi(\mu))=0$ the hypothesis $m_{1}(\mu)<\infty$ is not sufficient to guarantee almost sure convergence to the boundary. On the other hand, in this case the random walk $\left(\Phi\left(R_{n}\right)\right)_{n}$ on $\mathbb{Z}$ is recurrent (see Spitzer [12]) and if $\left(R_{n} o\right)_{n}$ converges almost surely, then it converges to $\omega_{1} \omega_{2}\left(\left(R_{n}^{i} o_{i}\right)_{n}\right.$ does not converge to $x^{i} \in \mathbb{T}^{i}$ since this would imply that $R_{n}^{i} o_{i}=x^{i}$ for all sufficiently large $n$, nor does it converge to $\xi^{i} \in \partial^{*} \mathbb{T}^{i}$, since this would imply that $h_{i}\left(R_{n}^{i} o_{i}\right)$ tends to infinity). We give a sufficient condition for almost sure convergence in the driftfree case.

Theorem 5.2. If $m_{1}(\mu)<\infty$, MEAN $(\Phi(\mu))=0$ and the following two conditions hold

$$
\left\{\begin{array}{l}
\mathbb{E}\left(\left|o_{1} \wedge\left(X_{1}^{1}\right)^{-1} o_{1}\right| p^{\left|o_{1} \wedge X_{1}^{1} o_{1}\right|}\right)<\infty, \\
\mathbb{E}\left(\left|o_{2} \wedge\left(X_{1}^{2}\right)^{-1} o_{2}\right| q^{\left|o_{2} \wedge X_{1}^{2} o_{2}\right|}\right)<\infty,
\end{array}\right.
$$

where $X_{1}^{i}$ is the $\Gamma_{i}$-valued random variable $(i=1,2)$ induced by $X_{1}$, then $R_{n} o \rightarrow \omega_{1} \omega_{2}$ almost surely. This holds, in particular, if these two simpler conditions are both satisfied

$$
\left\{\begin{array}{l}
\mathbb{E}\left(p^{\left|X_{1}^{1}\right|}\right)=\int_{\Gamma_{1}} p^{d_{1}\left(o_{1}, \gamma_{1} o_{1}\right)} \mathrm{d} \mu_{1}\left(\gamma_{1}\right)<\infty \\
\mathbb{E}\left(q^{\left|X_{1}^{2}\right|}\right)=\int_{\Gamma_{2}} q^{d_{2}\left(o_{2}, \gamma_{2} o_{2}\right)} \mathrm{d} \mu_{2}\left(\gamma_{2}\right)<\infty
\end{array}\right.
$$

or if $X_{1}$ has bounded range. 
Proof. The fact that conditions 4 are sufficient to guarantee almost sure convergence to $\omega_{1} \omega_{2}$ is simply a corollary of the result of Cartwright, Kaimanovich and Woess [5] Theorem 2.c. The conditions 5 are particular cases.

\section{LAW OF LARGE NUMBERS}

Given a random walk on $\Gamma$ defined as in Section 4 and satisfying the hypotheses there stated, we can prove the following strong law of large numbers.

Theorem 6.1. If $m_{1}(\mu)<\infty$ then

almost surely and in $L^{1}$.

$$
\lim _{n \rightarrow \infty} \frac{1}{n}\left|R_{n}\right|=|\operatorname{MEAN}(\Phi(\mu))|
$$

Proof. We first observe that since $m_{1}(\mu)$ is finite, by Kingman's subadditive ergodic theorem [9], $\frac{1}{n}\left|R_{n}\right|$ converges almost surely and in $L^{1}$. Note that from $d_{i}\left(o_{i}, x_{i}\right) \geq\left|h_{i}\left(x_{i}\right)\right| i=1,2$ it follows that $d\left(o_{1} o_{2}, x_{1} x_{2}\right) \geq$ $\left|h_{1}\left(x_{1}\right)\right|$ and $m_{1}(\mu) \geq m_{1}(\Phi(\mu))$. Moreover from the hypothesis we get $m_{1}\left(\mu_{i}\right)<\infty, i=1,2$, and then we can apply [5], Theorem 4 both to $\left|R_{n}^{1}\right|$ and $\left|R_{n}^{2}\right|$ :

$$
\begin{aligned}
& \frac{1}{n}\left|R_{n}^{1}\right| \longrightarrow\left|\operatorname{MEAN}\left(\Phi_{1}\left(\mu_{1}\right)\right)\right|, \\
& \frac{1}{n}\left|R_{n}^{2}\right| \longrightarrow\left|\operatorname{MEAN}\left(\Phi_{2}\left(\mu_{2}\right)\right)\right|,
\end{aligned}
$$

almost surely and in $L^{1}$. Moreover, by the strong law of large numbers, since $\Phi\left(R_{n}\right)=\Phi\left(X_{1}\right)+\cdots+\Phi\left(X_{n}\right)$ is the sum of $n$ independent identically distributed random variables $\left|\Phi\left(R_{n}\right)\right| / n$ converges to $|\operatorname{MEAN}(\Phi(\mu))|$ almost surely. Then, applying Proposition 3.1,

$$
\frac{1}{n}\left|R_{n}\right|=\frac{1}{n}\left(\left|R_{n}^{1}\right|+\left|R_{n}^{2}\right|-\left|\Phi\left(R_{n}\right)\right|\right) \longrightarrow|\operatorname{MEAN}(\Phi(\mu))|
$$

almost surely, but this must be the $L^{1}$ limit too.

\section{Central limit theorem}

We prove a central limit theorem for random walks as defined in Section 4. We first deal with the case of nonzero mean drift.

Theorem 7.1. If $m_{1}(\mu)<\infty, \operatorname{MEAN}(\Phi(\mu)) \neq 0$ and $\operatorname{VAR}(\Phi(\mu))<\infty$ then

$$
\frac{\left|R_{n}\right|-n|\operatorname{MEAN}(\Phi(\mu))|}{\sqrt{n \operatorname{VAR}(\Phi(\mu))}} \longrightarrow \mathcal{N}(0,1)
$$

where $\mathcal{N}(0,1)$ is the standard normal distribution and the limit is in law.

Proof. Let $\operatorname{MEAN}(\Phi(\mu))>0$. Then by Theorem $5.1, R_{n} o \rightarrow\left(R_{\infty}^{1}, \omega_{2}\right)$ almost surely and

$$
o_{1} \wedge R_{n}^{1} o_{1} \longrightarrow o_{1} \wedge R_{\infty}^{1}
$$

almost surely, where $o_{1} \wedge R_{\infty}^{1}$ is a random vertex on $\pi\left(o_{1}, \omega_{1}\right)$. Hence

$$
d_{1}\left(o_{1}, R_{n}^{1}\right)=\Phi_{1}\left(R_{n}^{1} o_{1}\right)+2\left|o_{1} \wedge R_{\infty}^{1}\right|
$$

with probability one for all but finitely many $n$. Then, using Proposition 3.1,

$$
\begin{aligned}
\left|R_{n}\right| & =d\left(o, R_{n} o\right)=\Phi\left(R_{n}\right)+2\left|o_{1} \wedge R_{\infty}\right|+d_{2}\left(o_{2}, R_{n}^{2} o_{2}\right)-\Phi\left(R_{n}\right) \\
& =2\left|o_{1} \wedge R_{\infty}\right|+d_{2}\left(o_{2}, R_{n}^{2} o_{2}\right)
\end{aligned}
$$

with probability one for all but finitely many $n$. Moreover, $\left|o_{1} \wedge R_{\infty}^{1}\right| / \sqrt{n} \rightarrow 0$ almost surely. Hence we only have to estimate

$$
\frac{d_{2}\left(o_{2}, R_{n}^{2} o_{2}\right)-n|\operatorname{MEAN}(\Phi(\mu))|}{\sqrt{n \operatorname{VAR}(\Phi(\mu))}}=\frac{d_{2}\left(o_{2}, R_{n}^{2} o_{2}\right)-n\left|\operatorname{MEAN}\left(\Phi_{2}\left(\mu_{2}\right)\right)\right|}{\sqrt{n \operatorname{VAR}\left(\Phi_{2}\left(\mu_{2}\right)\right)}}
$$


but this is the case of the random walk on $\operatorname{AFF}\left(\mathbb{T}_{q}\right)$, studied in [5] Theorem 6 , which states that the limit in law is the standard normal distribution.

The case MEAN $(\Phi(\mu))<0$ is completely analogous.

To study the driftfree case $(\operatorname{MEAN}(\Phi(\mu))=0)$ we shall need finiteness of $m_{2+\varepsilon}(\mu)$ for some $\varepsilon>0$ and some further notations.

Suppose that MEAN $(\Phi(\mu))=0$ and $\operatorname{VAR}(\Phi(\mu))<\infty$ (these two quantities cannot be both equal to zero by the assumption on non-exceptionality). Let

$$
\begin{aligned}
& \bar{M}_{n}=\max \left\{\Phi\left(R_{k}\right): k=0, \ldots, n\right\}, \\
& \underline{M}_{n}=\min \left\{\Phi\left(R_{k}\right): k=0, \ldots, n\right\},
\end{aligned}
$$

and let $\bar{M}_{n}^{i}, \underline{M}_{n}^{i}, i=1,2$ be the corresponding random variables induced by $\Phi_{i}, i=1,2$. By duality (see e. g. Feller $[7])$,

$$
\Phi\left(R_{n}\right)-\underline{M}_{n} \stackrel{\text { in law }}{=} \bar{M}_{n}
$$

and (see Billingsley [3], (11.2) and (11.1))

$$
\frac{1}{\sqrt{n \operatorname{VAR}(\Phi(\mu))}}\left(\Phi\left(R_{n}\right), \bar{M}_{n}\right) \rightarrow(U, V) \quad \text { in law },
$$

where $(U, V)$ is an $\mathbb{R}^{2}$-valued random variable whose distribution has density

$$
f(u, v)=\sqrt{\frac{2}{\pi}}(2 v-u) e^{-(2 v-u)^{2} / 2} 1_{\{v \geq \max \{0, u\}\}}(u, v) .
$$

Theorem 7.2. If $m_{2+\varepsilon}(\mu)<\infty$ for some $\varepsilon>0$ and $\operatorname{MEAN}(\Phi(\mu))=0$, then

$$
\frac{\left|R_{n}\right|}{\sqrt{n \operatorname{VAR}(\Phi(\mu))}} \longrightarrow 4 V-|U|-2 U
$$

in law, and the density of the random variable $4 V-|U|-2 U$ is:

$$
f_{4 V-|U|-2 U}(t)=\frac{1}{\sqrt{2 \pi}}\left(e^{-t^{2} / 4}-e^{-t^{2}}\right) 1_{t \geq 0}(t) .
$$

Proof. Let

$$
T_{i}(n)=\max \left\{k \in\{0, \ldots, n\}: \Phi_{i}\left(R_{k}\right)=\underline{M}_{n}\right\}
$$

Note that

$$
\left|R_{n}^{i}\right|=d_{i}\left(o_{i}, R_{n}^{i} o_{i}\right)=d_{i}\left(\left(R_{T_{i}(n)}^{i}\right)^{-1} o_{i},\left(R_{T_{i}(n)}^{i}\right)^{-1} R_{n}^{i} o_{i}\right),
$$

since $d_{i}(x, y)=d_{i}(\gamma x, \gamma y)$ for every $\gamma \in \Gamma_{i}, i=1,2$.

It can be easily seen that

$$
d_{i}(x, y)=d_{i}(x, x \wedge y)+d_{i}(x \wedge y, y)=h_{i}(x)+h_{i}(y)-2 h_{i}(x \wedge y), \quad i=1,2,
$$

hence

$$
\begin{aligned}
\left|R_{n}^{i}\right| & =h_{i}\left(\left(R_{T_{i}(n)}^{i}\right)^{-1} o_{i}\right)+h_{i}\left(\widetilde{R}_{T_{i}(n)}^{i} o_{i}\right)-2 h_{i}\left(\left(R_{T_{i}(n)}^{i}\right)^{-1} o_{i} \wedge \widetilde{R}_{T_{i}(n)}^{i} o_{i}\right) \\
& =\Phi_{i}\left(\left(R_{T_{i}(n)}^{i}\right)^{-1}\right)+\Phi_{i}\left(\widetilde{R}_{T_{i}(n)}^{i}\right)-2 h_{i}\left(\left(R_{T_{i}(n)}^{i}\right)^{-1} o_{i} \wedge \widetilde{R}_{T_{i}(n)}^{i} o_{i}\right) \\
& =-\Phi_{i}\left(R_{T_{i}(n)}^{i}\right)-\Phi_{i}\left(R_{T_{i}(n)}^{i}\right)+\Phi_{i}\left(R_{n}^{i}\right)-2 h_{i}\left(\left(R_{T_{i}(n)}^{i}\right)^{-1} o_{i} \wedge \widetilde{R}_{T_{i}(n)}^{i} o_{i}\right) \\
& =\Phi_{i}\left(R_{i}^{n}\right)-2 \underline{M}_{n}^{i}-2 h_{i}\left(\left(R_{T_{i}(n)}^{i}\right)^{-1} o_{i} \wedge \widetilde{R}_{T_{i}(n)}^{i} o_{i}\right)
\end{aligned}
$$

where $\widetilde{R}_{T_{i}(n)}^{i}:=\left(R_{T_{i}(n)}^{i}\right)^{-1} R_{n}^{i}$ (note that we used the definition of $T_{i}(n)$ and the fact that $\Phi_{i}$ are group homomorphisms). 
Moreover, let us note that, since $\Phi_{1}\left(R_{n}^{1}\right)=-\Phi_{2}\left(R_{n}^{2}\right),-\underline{M}_{n}^{2}=\bar{M}_{n}^{1}$. Hence, using Proposition 3.1 and equation 8 ,

$$
\begin{aligned}
\left|R_{n}\right|= & \Phi_{1}\left(R_{n}^{1}\right)-2 \underline{M}_{n}^{1}-2 h_{1}\left(\left(R_{T_{1}(n)}^{1}\right)^{-1} o_{1} \wedge \widetilde{R}_{T_{1}(n)}^{1} o_{1}\right) \\
& +\Phi_{2}\left(R_{n}^{2}\right)-2 \underline{M}_{n}^{2}-2 h_{2}\left(\left(R_{T_{2}(n)}^{2}\right)^{-1} o_{2} \wedge \widetilde{R}_{T_{2}(n)}^{2} o_{2}\right)-\left|\Phi\left(R_{n}\right)\right| \\
= & 2\left(\bar{M}_{n}^{1}-\underline{M}_{n}^{1}\right)-\left|\Phi\left(R_{n}\right)\right|-2 h_{1}\left(\left(R_{T_{1}(n)}^{1}\right)^{-1} o_{1} \wedge \widetilde{R}_{T_{1}(n)}^{1} o_{1}\right) \\
& -2 h_{2}\left(\left(R_{T_{2}(n)}^{2}\right)^{-1} o_{2} \wedge \widetilde{R}_{T_{2}(n)}^{2} o_{2}\right) \\
= & 2 \bar{M}_{n}^{1}+2\left(\Phi_{1}\left(R_{n}^{1}\right)-\underline{M}_{n}^{1}\right)-2 \Phi_{1}\left(R_{n}^{1}\right)-\left|\Phi\left(R_{n}\right)\right|-2 h_{1}\left(\left(R_{T_{1}(n)}^{1}\right)^{-1} o_{1} \wedge \widetilde{R}_{T_{1}(n)}^{1} o_{1}\right) \\
& -2 h_{2}\left(\left(R_{T_{2}(n)}^{2}\right)^{-1} o_{2} \wedge \widetilde{R}_{T_{2}(n)}^{2} o_{2}\right) \\
& 2 \bar{M}_{n}^{1}+2\left(\Phi_{1}\left(R_{n}^{1}\right)-\underline{M}_{n}^{1}\right)-2 \Phi\left(R_{n}\right)-\left|\Phi\left(R_{n}\right)\right|-2 h_{1}\left(\left(R_{T_{1}(n)}^{1}\right)^{-1} o_{1} \wedge \widetilde{R}_{T_{1}(n)}^{1} o_{1}\right) \\
& -2 h_{2}\left(\left(R_{T_{2}(n)}^{2}\right)^{-1} o_{2} \wedge \widetilde{R}_{T_{2}(n)}^{2} o_{2}\right) .
\end{aligned}
$$

By equation 6, the last term is equal in law to

$$
4 \bar{M}_{n}^{1}-2 \Phi\left(R_{n}\right)-\left|\Phi\left(R_{n}\right)\right|-2 h_{1}\left(\left(R_{T_{1}(n)}^{1}\right)^{-1} o_{1} \wedge R_{T_{1}(n), n}^{1} o_{1}\right)-2 h_{2}\left(\left(R_{T_{2}(n)}^{2}\right)^{-1} o_{2} \wedge R_{T_{2}(n), n}^{2} o_{2}\right) .
$$

Now, [5] Proposition 3 shows that

$$
\frac{1}{\sqrt{n}} h_{i}\left(\left(R_{T_{i}(n)}^{i}\right)^{-1} o_{i} \wedge R_{T_{i}(n), n}^{i} o_{i}\right) \rightarrow 0
$$

in probability (hence in law), then, as $n \rightarrow \infty,\left|R_{n}\right| / \sqrt{n \operatorname{VAR}(\Phi(\mu))}$ behaves in law as

$$
\frac{4 \bar{M}_{n}^{1}-\left|\Phi_{1}\left(R_{n}^{1}\right)\right|-2 \Phi_{1}\left(R_{n}^{1}\right)}{\sqrt{n \operatorname{VAR}(\Phi(\mu))}}=\frac{4 \bar{M}_{n}-\left|\Phi\left(R_{n}\right)\right|-2 \Phi\left(R_{n}\right)}{\sqrt{n \operatorname{VAR}(\Phi(\mu))}},
$$

where equality holds both in law and almost surely. The result now follows from 7 , while the density is easily computed.

\section{AsYmptotic TYPE}

In the preceding sections we studied a large family of random processes on $D L(p, q)$ and then showed that irreducible, $\Gamma$-invariant random walks are elements of this family. Now we follow a somehow reversed path: we study the asymptotic type of the simple random walk on $D L(p, q)$ and then observe that it is the same asymptotic type of more general random walks.

We recall the definitions of the asymptotic type of a numerical sequence and of a random walk.

Definition 8.1. Given two non-negative sequences $\left(a_{n}\right)_{n}$ and $\left(b_{n}\right)_{n}$, we say that $a_{n} \preceq b_{n}$ if there are $C \geq c>0$ such that for all sufficiently large $n, a_{n} \leq C \sup \left\{b_{k}: c n \leq k \leq C n\right\}$; and $a_{n}$ is of the same asymptotic type of $b_{n}$ (and we write $a_{n} \approx b_{n}$ ) if $a_{n} \preceq b_{n}$ and $b_{n} \preceq a_{n}$.

Note that if $a_{n} \stackrel{n}{\sim} b_{n}$ then also $a_{n} \approx b_{n}$. Moreover, the definition can be simplified if the sequences are monotone: if, for instance, $\left(b_{n}\right)_{n}$ is non-increasing, then $a_{n} \preceq b_{n}$ if there are $C \geq c>0$ such that

for sufficiently large $n$.

$$
a_{n} \leq C b_{[c n]+1}
$$

Definition 8.2. The asymptotic type of a random walk $(X, P)$ is the asymptotic type of the sequence $\left(p^{(n d)}(x, x)\right)_{n}$, where $d=\operatorname{HCF}\left\{n: p^{(n)}(x, x)>0\right\}$ is the period (HCF denotes the highest common factor).

Note that by irreducibility the asymptotic type does not depend on the particular choice of $x$. Indeed, let $x, y \in X$, then there exist $n_{1}, n_{2} \in \mathbb{N}$ such that $p^{\left(n_{1}\right)}(x, y)>0$ and $p^{\left(n_{2}\right)}(y, x)>0$. If $n$ is a multiple of $d$ (note that $n_{1}+n_{2}$ is surely a multiple of $d$ )

$$
p^{\left(n_{2}\right)}(y, x) \cdot p^{(n)}(x, x) \cdot p^{\left(n_{1}\right)}(x, y) \leq p^{\left(n+n_{1}+n_{2}\right)}(y, y) .
$$


Hence if $C=\max \left\{2,1 /\left(p^{\left(n_{1}\right)}(x, y) \cdot p^{\left(n_{2}\right)}(y, x)\right)\right\}, c=1$ and $n \geq n_{1}+n_{2}$, we have that

$$
p^{(n)}(x, x) \leq C \sup \left\{p^{(k)}(y, y): k \equiv 0 \bmod d, c n \leq k \leq C n\right\}
$$

(note that $n \leq n+n_{1}+n_{2} \leq 2 n \leq C n$ ).

In the case of the simple random walk on $D L(p, q)$ we have of course $p^{(n)}(x, x)=p^{(n)}(y, y)$ for all $x, y$, since these graphs are vertex transitive, and $d=2$, whence $p^{(2 n+1)}(x, x)=0$ for every $n \in \mathbb{N}$. Moreover, $\left(p^{(2 n)}(x, x)\right)_{n}$ is a monotone non increasing sequence, namely the following proposition holds (in the case of random walks on groups this fact was stated by Avez [2]).

Proposition 8.3. If $(X, P)$ is a symmetric random walk then for all $x \in X$ we have

$$
p^{(2 n+2)}(x, x) \leq p^{(2 n)}(x, x) .
$$

Proof. By Hölder inequality and the symmetry of the random walk,

$$
\begin{aligned}
p^{(2 n+2)}(x, x) & =\sum_{y \in X} p^{(n)}(x, y) p^{(n+2)}(y, x) \\
& \leq \sqrt{\sum_{y \in X}\left(p^{(n)}(x, y)\right)^{2} \sum_{y \in X}\left(p^{(n+2)}(x, y)\right)^{2}} \\
& =\sqrt{p^{(2 n)}(x, x) p^{(2 n+2)}(x, x)} .
\end{aligned}
$$

Moreover, $p^{(2 n)}(x, x) \geq\left(p^{(2)}(x, x)\right)^{n}>0$, hence $p^{(2 n+2)}(x, x) / p^{(2 n)}(x, x)$ is a nondecreasing function of $n$. If this ratio were ever strictly greater than 1 then $p^{(2 n)}(x, x)$ would go to infinity. Hence, it is always less than or equal to 1 .

If $p \neq q$ the asymptotic type of the simple random walk on $D L(p, q)$ (indeed, of any symmetric random walk with finite range) is already known to be that of $e^{-n}$. In fact the asymptotic type of symmetric random walks with finite range on non-amenable graphs is $\left(e^{-n}\right)_{n}$ (see [14] Paragraph 15), and $D L(p, q)$ is amenable if and only if $p=q$ (see [14], (12.18)).

Therefore we are interested in determining the asymptotic type of the simple random walk on $D L(p, p)$. Since $D L(p, q)$ has exponential growth (even when $p=q$ ), we have the following inequality for the transition probabilities of the simple random walk:

$$
p^{(2 n)}(x, x) \leq C_{1} \exp \left(-C_{2} n^{1 / 3}\right),
$$

for some $C_{1}, C_{2}>0$, for all $n \in \mathbb{N}$ and for all $x \in D L(p, p)$ (see Saloff-Coste [11]). Then if we prove the following theorem it will be clear that the asymptotic type we try to determine is $\left(e^{-n^{1 / 3}}\right)_{n}$.

Theorem 8.4. If $p^{(n)}(x, x)$ are the transition probabilities of the simple random walk on $D L(p, p)$, then there are costants $C, D>0$ such that

$$
p^{(2 n)}(x, x) \geq C \exp \left(-D n^{1 / 3}\right) \quad \text { for all } n, \text { for all } x \text {. }
$$

Corollary 8.5. The asymptotic type of the simple random walk on $D L(p, p)$ is $\left(e^{-n^{1 / 3}}\right)_{n}$.

Let us observe that, by a result of Pittet and Saloff-Coste [10], given a vertex-transitive graph and two transitive transition matrices $P_{1}$ and $P_{2}$ (that is $p_{i}(x, y)=p_{i}(\gamma x, \gamma y)$ for all $\gamma \in \operatorname{AUT}(X)$, for $\left.i=1,2\right)$, and with finite second moment, the asymptotic type of $p_{1}^{(n d)}(x, x)$ is the same as that of $p_{2}^{(n d)}(x, x)$ (see also [14]).

Corollary 8.6. The asymptotic type of a transitive random walk on $D L(p, q)$ with finite second moment is $\left(e^{-n}\right)_{n}$ if $p \neq q$ and $\left(e^{-n^{1 / 3}}\right)_{n}$ if $p=q$.

In order to prove Theorem 8.4 we need a lemma and a choice of coordinates on $D L(p, p)$. To a given point $x=x_{1} x_{2} \in D L(p, p)$ we associate three coordinates: $a(x):=d_{1}\left(x_{1}, o_{1}\right), b(x):=d_{2}\left(x_{2}, o_{2}\right), c(x):=h_{1}\left(x_{1}\right)$. We note that if $\left(Z_{n}\right)_{n}$ is the sequence of random variables which represents the simple random walk on 
$D L(p, p)$ then $\left(c\left(Z_{n}\right)\right)_{n}$ is a sequence of integer valued random variables which turns out to represent the simple random walk on $\mathbb{Z}$. Indeed, the mapping

$$
\begin{array}{cl}
\widetilde{\Phi}: & D L(p, p) \mapsto \mathbb{Z} \\
& x_{1} x_{2} \mapsto h_{1}\left(x_{1}\right)
\end{array}
$$

induces a projection of any random walk on $D L(p, p)$ onto a random walk on $\mathbb{Z}$. Since the distribution of $Z_{0}$ does not affect the asymptotic type of a random walk, for simplicity we will suppose that $Z_{0}=o_{1} o_{2}$ (whence $\left.c\left(Z_{0}\right)=0\right)$.

Lemma 8.7. Let $r, m \in \mathbb{N}$ and let

$$
A_{m, r}:=\{x \in D L(p, p): a(x) \leq r, b(x) \leq r,|c(x)| \leq m\} .
$$

Then

(a) if $r \leq m$ then $\left|A_{m, r}\right|=p^{r-1}(r+p(r+1))$,

(b) if $r>m$ then

$$
\left|A_{m, r}\right|= \begin{cases}p^{r}(1+m)+m p^{r-1} & \text { if } r \text { and } m \text { have the same parity } \\ p^{r} m+(m+1) p^{r-1} & \text { otherwise. }\end{cases}
$$

Proof. (a) If $r \leq m$, then $A_{m, r}=\{x \in D L(p, p): a(x) \leq r, b(x) \leq r\}$, since $\left|h_{1}\left(x_{1}\right)\right| \leq d_{1}\left(o_{1}, x_{1}\right)$. In order to distinguish between the horocycles of $\mathbb{T}^{1}$ and the ones of $\mathbb{T}^{2}$, we put $H_{j}^{i}$ for the $j$-th horocycle of $\mathbb{T}^{i}$, $i=1,2$. Moreover, we define

$$
H_{j, r}^{i}:=\left\{x \in H_{j}^{i}: d_{i}\left(o_{i}, x\right) \leq r\right\}, \quad i=1,2 .
$$

Then, if $r \leq m, A_{m, r}$ consists of all the points $x_{1} x_{2} \in D L(p, p)$ such that $x_{1} \in H_{j, r}^{1}, x_{2} \in H_{-j, r}^{2},|j| \leq r$. From that we get the following expression for $\left|A_{m, r}\right|$ :

$$
\left|A_{m, r}\right|=\left|H_{0, r}^{1}\right|^{2}+2 \sum_{j=1}^{r}\left|H_{j, r}^{1}\right| \cdot\left|H_{-j, r}^{1}\right|
$$

where in the second equality we used the fact that the degree of $\mathbb{T}^{1}$ is the same as the degree of $\mathbb{T}^{2}$. Then all we have to do is count the elements of $H_{j, r}^{1}$ for $|j| \leq r$.

We note that $d_{1}\left(o_{1}, x\right)=d_{1}\left(x, x \wedge o_{1}\right)+d_{1}\left(o_{1}, x \wedge o_{1}\right)$ for all $x \in \mathbb{T}^{1}$, then if we let $k(x)=d_{1}\left(x, x \wedge o_{1}\right)$ and $l(x)=d_{1}\left(o_{1}, x \wedge o_{1}\right)$,

$$
\begin{aligned}
d_{1}\left(o_{1}, x\right) & =k(x)+l(x), \\
h_{1}(x) & =k(x)-l(x) .
\end{aligned}
$$

Then $H_{j, r}^{1}$ consists of all $x \in \mathbb{T}^{1}$ such that $k(x)-l(x)=j$ and $k(x)+l(x) \leq r$.

We claim that $\left|H_{j, r}^{1}\right|=p^{\left[\frac{r+j}{2}\right]}$. Indeed, if $k, l \in \mathbb{N}$ are such that $k+l \leq r$ and $k-l=j$, then all of the $k$-th descendants of the origin's $l$-th ancestor (which are exactly $p^{k}$ ) are elements of $H_{j, r}^{1}$. Let $B(k, l)$ be the set of these $k$-th descendants of the $l$-th ancestor of the origin, then $B(k+1, l+1) \supset B(k, l)$ and still $(k+1)-(l+1)=j$. Moreover if $\bar{k}=\max \{k \in \mathbb{N}: k+l \leq r, k-l=j\}$ and $\bar{l}$ is the corresponding value of $l(\bar{l}=\bar{k}-j)$, then $\bar{k}=\left[\frac{r+j}{2}\right]$ and $H_{j, r}^{1}=B(\bar{k}, \bar{l})$. Hence $\left|H_{j, r}^{1}\right|=p^{\left[\frac{r+j}{2}\right]}$ and

$$
\left|A_{m, r}\right|=p^{2\left[\frac{r}{2}\right]}+2 \sum_{j=1}^{r} p^{\left[\frac{r+j}{2}\right]} p^{\left[\frac{r-j}{2}\right]}
$$

It is easy to see that whatever the parity of $r$ is,

$$
\left|A_{m, r}\right|=p^{r-1}(r+(r+1) p) .
$$


(b) If $r>m$, the computation of $\left|A_{m, r}\right|$ will deal only with $H_{j, r}^{1}$ with $|j| \leq m$, namely

$$
\begin{aligned}
\left|A_{m, r}\right| & =\left|H_{0, r}^{1}\right|^{2}+2 \sum_{j=1}^{m}\left|H_{j, r}^{1}\right| \cdot\left|H_{-j, r}^{1}\right| \\
& =p^{2\left[\frac{r}{2}\right]}+2 \sum_{j=1}^{m} p^{\left[\frac{r+j}{2}\right]} p^{\left[\frac{r-j}{2}\right]} .
\end{aligned}
$$

Then we distinguish two cases: it is easy to show that

(i) if $r$ and $m$ have the same parity, then

$$
\left|A_{m, r}\right|=p^{r-1}(m+(m+1) p) .
$$

(ii) if $r$ and $m$ do not have the same parity, then:

$$
\left|A_{m, r}\right|=p^{r-1}(m+1+m p) .
$$

We also state a particular case of Lemma 1.2 of Alexopoulos [1], which will be needed in the proof of Theorem 8.4.

Lemma 8.8. Let $\left(S_{n}\right)_{n}$ be the sequence of random variables which represents the simple random walk on $\mathbb{Z}$ with $S_{0}=0$. Moreover, let $M_{n}:=\max _{1 \leq i \leq n}\left|S_{i}\right|$ for $n \geq 1$. Then there are positive constants $c_{1}, c_{2}$, $m_{0}, n_{0} \in \mathbb{N}$ such that for all integers $n \geq n_{0}, m \geq m_{0}$ we have

$$
\mathbb{P}\left[M_{n} \leq m\right] \geq c_{1} e^{-c_{2} n / m^{2}} .
$$

Now we are ready to prove Theorem 8.4.

Proof of Theorem 8.4. Let $x=o, A$ be a subset of $D L(p, p)$ and $Z_{n}$ be the random position of the walk in $X$ at time $n$, using the Cauchy-Schwarz inequality (and the symmetry of the simple random walk) we obtain:

$$
p^{(2 n)}(o, o) \geq \sum_{y \in A} p^{(n)}(o, y)^{2} \geq\left(\sum_{y \in A} p^{(n)}(o, y)\right)^{2} \frac{1}{|A|}=\mathbb{P}_{o}\left[Z_{n} \in A\right]^{2} \frac{1}{|A|} .
$$

Then the proof consists in finding a suitable family of sets among which we choose $A$ ( $A$ will depend on $n$ ). Consider the family of sets

$$
A_{m}:=A_{m, 3 m}=\{x \in D L(p, p): a(x) \leq 3 m, b(x) \leq 3 m,|c(x)| \leq m\} .
$$

We denote $\left(c\left(Z_{n}\right)\right)_{n}$ by $\left(S_{n}\right)_{n}:\left(S_{n}\right)_{n}$ satisfies the hypothesis of Lemma 8.8, and we let $M_{n}$ be defined as in this lemma.

If $M_{n} \leq m$, then $a\left(Z_{n}\right) \leq 3 m$ and $b\left(Z_{n}\right) \leq 3 m$, hence

$$
\mathbb{P}_{o}\left[Z_{n} \in A_{m}\right] \geq \mathbb{P}_{o}\left[M_{n} \leq m\right]
$$

Moreover, applying Lemma 8.8,

$$
\mathbb{P}_{o}\left[M_{n} \leq m\right] \geq c_{1} \exp \left(-c_{2} \frac{n}{m^{2}}\right)
$$

for $n$ and $m$ sufficiently large and for some $c_{1}, c_{2}>0$.

Now we choose $m=\left[n^{1 / 3}\right]$ (where [.] denotes the integer part), and estimate $\left|A_{m}\right|$ : by Lemma 8.7

$$
\left|A_{m}\right|= \begin{cases}p^{\left[3 n^{1 / 3}\right]}\left(1+\left[n^{1 / 3}\right]\right)+\left[n^{1 / 3}\right] p^{\left[3 n^{1 / 3}\right]-1} & \text { if }\left[3 n^{1 / 3}\right] \text { and }\left[n^{1 / 3}\right] \text { have the same parity } \\ p^{\left[3 n^{1 / 3}\right]}\left[n^{1 / 3}\right]+\left(\left[n^{1 / 3}\right]+1\right) p^{\left[n^{1 / 3}\right]-1} & \text { otherwise. }\end{cases}
$$

In both cases

for some $c_{3}>0$. Hence

$$
\left|A_{m}\right| \leq p^{3 n^{1 / 3}}\left(1+2 n^{1 / 3}\right) \leq 3 n^{1 / 3} p^{3 n^{1 / 3}} \leq \exp \left(c_{3}\left(1+\log (n)+n^{1 / 3}\right)\right)
$$

$$
\frac{\mathbb{P}_{o}\left[Z_{n} \in A_{n^{1 / 3}}\right]^{2}}{\left|A_{n^{1 / 3}}\right|} \geq \frac{c_{1}^{2} \exp \left(-2 c_{2} n^{1 / 3}\right)}{\exp \left(c_{3}\left(1+\log (n)+n^{1 / 3}\right)\right)}
$$


which leads to the conclusion.

Acknowledgement The author would like to thank Wolfgang Woess for suggesting the subject of this paper.

\section{REFERENCES}

[1] G. Alexopoulos, A lower estimate for central probabilities on polycyclic groups, Can. J. Math. 44 (5) (1992), 897-910.

[2] A. Avez, Limite des quotients pour des marches aléatoires sur des groupes, C. R. Acad. Sci. Paris Sér. A 276 (1973), 317-320.

[3] P. Billingsley, Convergence of probability measures, Wiley, New York, 1968.

[4] P. Cartier, Fonctions harmoniques sur un arbre, Symposia Math. 9 (1972), 203-270.

[5] D. I. Cartwright, V. A. Kaimanovich, W. Woess, Random walks on the affine group of local fields and of homogeneous trees, Ann. Inst. Fourier (Grenoble) 44 (1994), 1243-1288.

[6] R. Diestel, I. Leader, A conjecture concerning a limit of non-Cayley graphs, preprint.

[7] W. Feller, An introduction to probability theory and its applications, Vol II, Wiley, New York, 1966.

[8] E. Hewitt, K. A. Ross, Abstract Harmonic Analysis, Vol. I, Springer-Verlag, Berlin-Heidelberg-New York, 1963.

[9] J. F. C. Kingman, The ergodic theory of subadditive processes, J. Royal Stat. Soc., Ser. B 30 (1968), 499-510.

[10] Ch. Pittet, L. Saloff-Coste, On the stability of the behaviour of random walks on groups, J.Geometric Anal., in press.

[11] L. Saloff-Coste, Isoperimetric inequalities and decay of iterated kernels for almost-transitive graphs, Combinatorics, Probability and Computing 4 (1995), 419-442.

[12] F. Spitzer, Principles of Random Walks, Springer, New York, 1976.

[13] W. Woess, Boundaries of random walks on graphs and groups with infinitely many ends, Israel J. Math. 68 (1989), 271-301.

[14] W. Woess, Random walks on infinite graphs and groups, Cambridge Tracts in Mathematics 138, Cambridge Univ. Press, 2000.

D. Bertacchi, Università di Milano-Bicocca Dipartimento di Matematica e Applicazioni, Via Bicocca degli Arcimboldi 8, 20126 Milano, Italy.

E-mail address: bertacchi@matapp.unimib.it 